\title{
Alveolar Soft Part Sarcoma of the Extremity: Case Report and Literature Review
}

\author{
Khaled J. Ata ${ }^{\mathrm{a}, \mathrm{d}}$, Hani N. Farsakh ${ }^{\mathrm{a}}$, Anwar Rjoop ${ }^{\mathrm{b}}$, \\ Ismail Matalka ${ }^{b}$, Liqa A. Rousan ${ }^{c}$
}

\begin{abstract}
Alveolar soft part sarcoma (ASPS), a rare soft tissue sarcoma in children and adolescents, carries a poor prognosis. ASPS is an aggressive tumor of controversial histogenesis that, unlike other soft tissue sarcomas, tends to metastasize to the brain. A 9-year-old boy presented to our outpatient clinic in April 2009 with a chief complaint of a large painless mass in the left thigh whose size had increased significantly over the past 10 months. After staging the tumor, we performed open biopsy; the diagnosis was ASPS and he underwent wide local excision. In the course of 4-year follow-up by clinical and imaging studies, there was no evidence of early tumor recurrence or metastasis. Complete surgical resection is the treatment of choice in patients with ASPS.
\end{abstract}

Keywords: Alveolar soft part sarcoma; Extremity sarcoma

\section{Introduction}

Soft tissue sarcoma is a type of malignant soft tissue tumor that is usually linked to the tissue of origin. In alveolar soft part sarcomas (ASPS), the alveolar description is based on the histological pattern of the tumor. ASPS, a rare, malignant soft tissue neoplasm that affects young patients, accounts for approximately $0.5-1.0 \%$ of all soft tissue sarcomas [1]

Manuscript accepted for publication February 5, 2014

${ }^{a}$ Department of Orthopedics, Jordan University of Science and Technology, King Abdullah University Hospital, Irbid, Jordan ${ }^{\mathrm{b}}$ Department of Pathology, Jordan University of Science and Technology, King Abdullah University Hospital, Irbid, Jordan ${ }^{c}$ Department of Radiology and Nuclear Medicine, Jordan University of Science and Technology, King Abdullah University Hospital, Irbid, Jordan

${ }^{\mathrm{d}}$ Corresponding author: Khaled J. Ata, Department of Orthopedics, School of Medicine, King Abdullah University Hospital, Jordan University of Science and Technology, PO Box 3030, Irbid 22110, Jordan. Email: drkata@yahoo.com

doi: http://dx.doi.org/10.14740/wjon777w and for $5.0 \%$ of pediatric non-rhabdomyosarcoma soft tissue sarcomas [2]. While it usually presents as a painless mass in the extremities, these tumors have been reported to arise in other parts of the body [3]. Compared to other sarcomas, they are characterized by an unusual pattern of metastatic spread; brain metastasis is a common feature [4].

\section{Case Report}

This 9-year-old boy presented to our outpatient clinic in April 2009 with a chief complaint of a painless large mass in the left thigh. He and his parents first noticed it as a small lump that increased in size over the next year till he visited our hospital. On examination, a mass measuring about $10 \mathrm{~cm}$ in diameter was noted at the anteromedial aspect of his left thigh about $15 \mathrm{~cm}$ above the knee joint. On palpation, it was firm and immobile. The mass was neither tender nor hot and there were no changes in the overlying skin (Fig. 1).

Magnetic resonance imaging (MRI) of the left thigh,

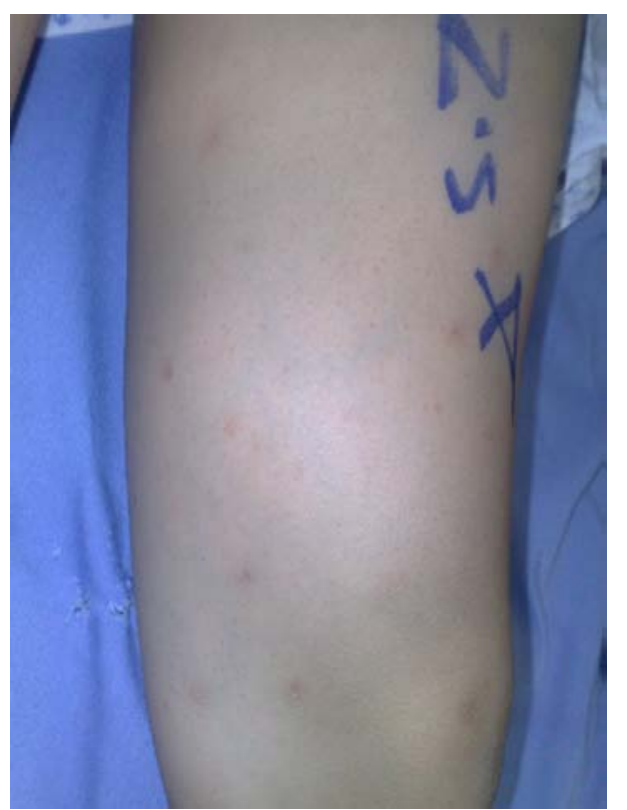

Figure 1. Palpable mass at inner aspect of distal thigh. 


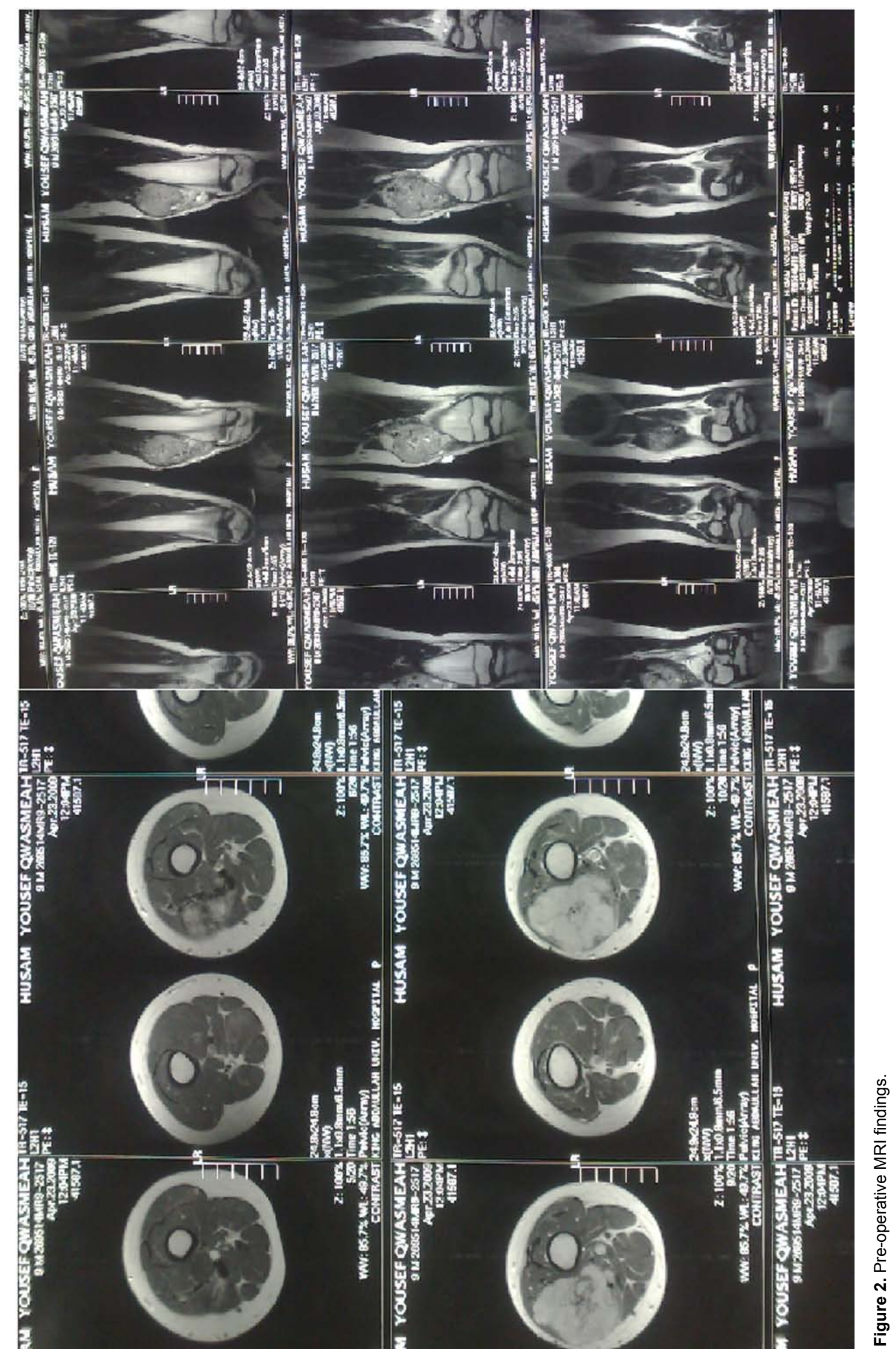



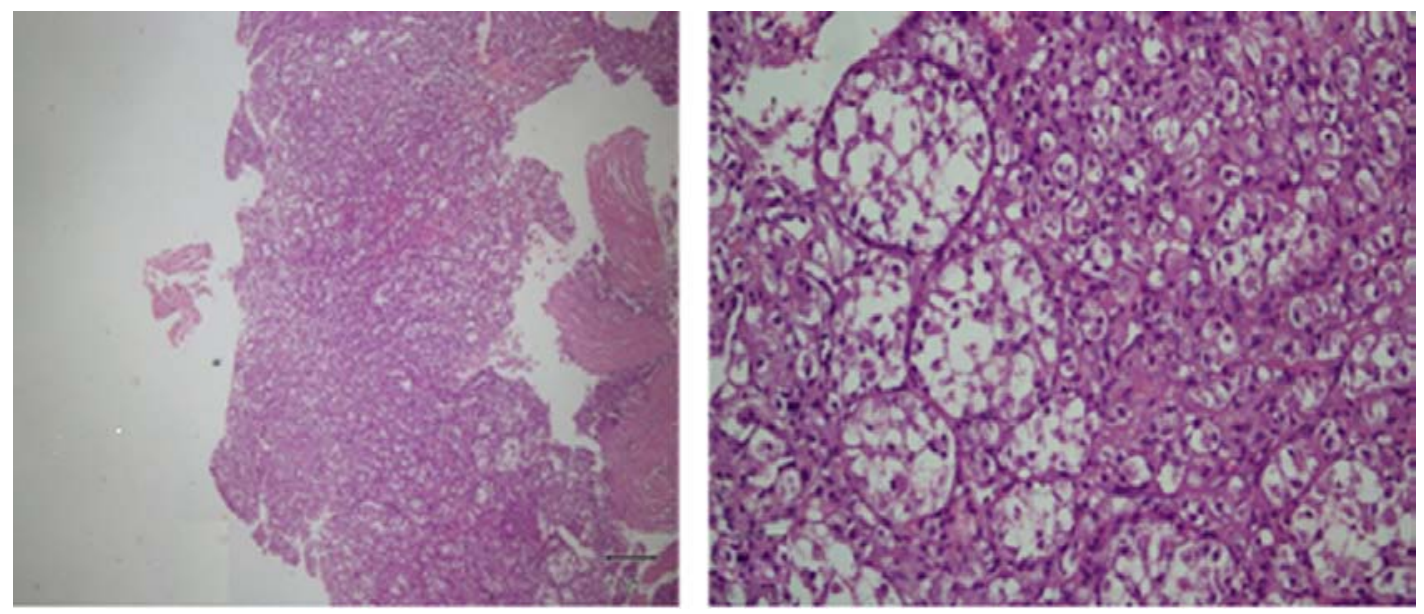

Figure 3. H\&E of the incisional biopsy shows nested pattern arrangement of malignant cells which are large, polygonal at higher magnification.

performed later in the same week, revealed a well-defined soft tissue mass, heterogeneously enhanced, measured $6.5 \times$ $4 \times 10 \mathrm{~cm}$, displaced and invaded the lower vastus medialis muscle. There was no involvement or invasion of underlying bones and no evidence of a periosteal reaction (Fig. 2). The superficial femoral vein and artery were displaced laterally.

These findings were suggestive of a heterogeneous soft tissue mass, possibly a sarcoma. Although a close biopsy is a realistic option, we did an open incisional biopsy, performed through a medial longitudinal incision centralized over the mass; it revealed multiple fragments of white soft tissue. Microscopic study showed sheets of malignant cells arranged in a nested pattern. They were large, polygonal with distinct cell borders and eosinophilic cytoplasm; they were separated by thin-walled vascular channels (Fig. 3).

The tumor cells contained intracellular crystals that were PAS-positive and diastase-resistant (Fig. 4). Immunostaining was negative for pan-cytokeratin, actin, desmin, myo- genin, chromogranin, HMB45, melan A, vimentin, inhibin and S100. Based on these findings, a diagnosis of ASPS was made.

On whole-body bone (Tc99m), enhanced chest, abdominal, pelvic computed tomography (CT) and brain MRI scans, there was no evidence of distant metastasis.

After complete radiological and histological studies, he underwent wide local excision via an elliptical surgical incision to include the scar and tract of the earlier biopsy. Then we identified major vessels and performed a wide resection taking care not to expose the tumor. On gross examination, the tumor was a $12 \times 8 \times 6 \mathrm{~cm}$ mass with an ellipse of skin on its surface (Fig. 5).

The results of microscopic examination were similar to those of biopsy. All surgical margins were free; the closest was located $4 \mathrm{~mm}$ medially.

The postoperative course was unremarkable. The patient was seen every $5-7$ months for clinical and radiological
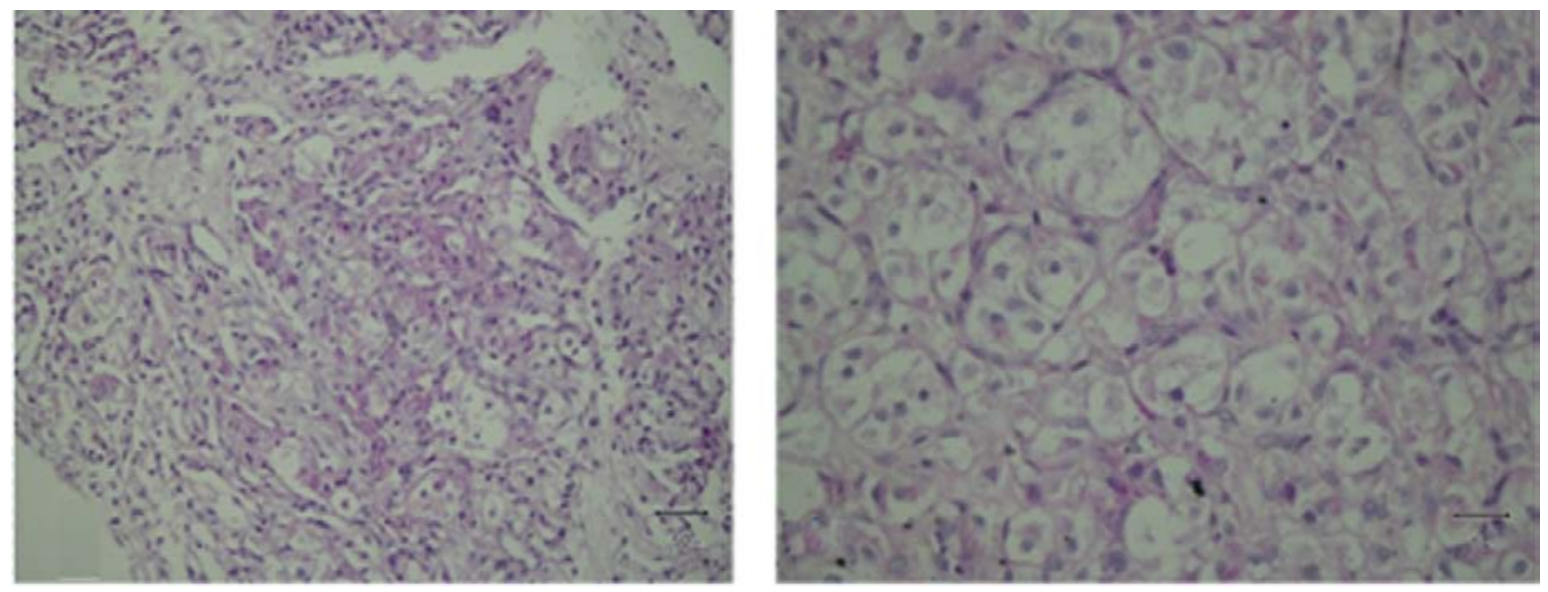

Figure 4. PAS/diastase stain positive for intracellular rod-shaped crystalloids. 


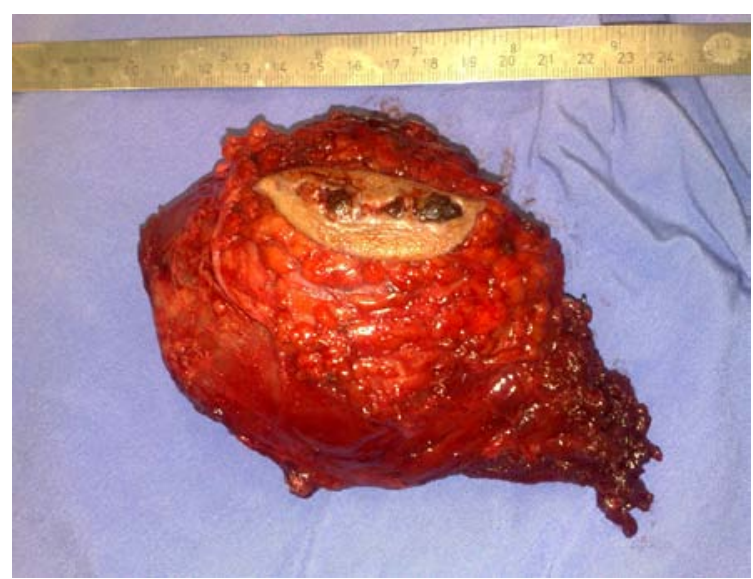

Figure 5. Soft tissue tumor after excision with biopsy tract.

follow-up by local and brain MRI, CT chest, abdominal and pelvic scans. In the course of 48 months, there have been no signs of recurrence or metastasis.

\section{Discussion}

ASPS, first described in 1952 by Christopherson et al [5], is characterized by an indolent course of slow growth and a high mortality risk. The tumor mainly affects the extremities with the thigh being the most common site although these tumors have been reported to arise in the trunk, the head and neck, and the tongue.

Metastasis usually occurs late in the course of the disease and involves the lungs, bones, lymph nodes and brain. Metastasis to the liver has also been reported [6]. To rule out brain metastasis, the Surgical Society of Oncology recommends intracranial imaging in all patients with ASPS [7].

There is some controversy over the pathogenesis of ASPS. While Mukai et al [8] provided support for a myogenic origin, Marchac et al [9] reported ASPL-TFE3 transcript fusion produced by chromosomal translocation (X; 17). According to Ordonez [10], there is a large female preponderance among ASPS patients. On the other hand, in their retrospective study, Portera et al [11] detected no significant gender difference when the patient population was considered as a whole although when they assessed patients with localized disease separately they also found a definite (64\%) female preponderance.

The histological characteristics of ASPS include organoid nests of polygonal tumor cells encompassed by a dense capillary vasculature. The cells are of uniform size and shape and contain granular, eosinophilic cytoplasm surrounding a vesicular nucleus that hosts a prominent nucleolus. The designation "alveolar" derives from the pseudo-alveolar appearance [12].

While the role of radio- and chemotherapy is yet to be established, surgical resection with margins microscopically free of tumor cells remains the ASPS treatment of choice and represents a strong indicator of the treatment outcome [13]. Spontaneous regression has been reported [14].

According to Lieberman et al [12] who followed 102 patients over 63 years, the survival rate of patients with no metastasis at the time of diagnosis decreased dramatically from $77 \%$ at 2 years to $60 \%$ at 5 years, $38 \%$ at 10 years and $15 \%$ at 20 years. This indicates that periodic follow-up is mandatory in all patients with ASPS.

\section{Conclusion}

In conclusion, ASPS is a rare type of sarcomas that affects primarily the lower limbs. Periodic radiologic follow-up is mandatory to detect metastasis. While the role of radio- and chemotherapy is yet to be established, complete surgical resection is the treatment of choice in patients with ASPS.

\section{Competing Interests}

The authors declare that they have no competing interests.

\section{References}

1. Enzinger FM, Weiss SW. Soft Tissue Tumors. St. Louis, MO: CV Mosby; Alveolar soft part sarcoma. 1995; 13:1067-1074.

2. Rao BN. Nonrhabdomyosarcoma in children: prognostic factors influencing survival. Semin Surg Oncol. 1993;9(6):524-531.

3. Kayton ML, Meyers P, Wexler LH, Gerald WL, LaQuaglia MP. Clinical presentation, treatment, and outcome of alveolar soft part sarcoma in children, adolescents, and young adults. J Pediatr Surg. 2006;41(1):187-193.

4. Perry JR, Bilbao JM. Metastatic alveolar soft part sarcoma presenting as a dural-based cerebral mass. Neurosurgery. 1994;34(1):168-170.

5. Christopherson WM, Foote FW, Jr., Stewart FW. Alveolar soft-part sarcomas; structurally characteristic tumors of uncertain histogenesis. Cancer. 1952;5(1):100-111.

6. Zhang RY. [The histogenesis of alveolar soft part sarcoma: a clinicopathological analysis of 135 cases]. Zhonghua Bing Li Xue Za Zhi. 1990;19(3):165-168.

7. Pollack R, Brennan M, Lawrence W, Jr. Society of Surgical Oncology practice guidelines. Soft-tissue sarcoma surgical practice guidelines. Oncology (Williston Park). 1997;11(9):1327-1332.

8. Mukai M, Torikata C, Iri H, Mikata A, Hanaoka H, Kato K, Kageyama K. Histogenesis of alveolar soft part sarcoma. An immunohistochemical and biochemical study. 
Am J Surg Pathol. 1986;10(3):212-218.

9. Marchac A, Picard A, Landman-Parker J, Larroquet M, Vazquez MP, Franchi G. [A pediatric case of Alveolar Soft Part Sarcoma]. Rev Stomatol Chir Maxillofac. 2007;108(6):547-550.

10. Ordonez NG. Alveolar soft part sarcoma: a review and update. Adv Anat Pathol. 1999;6(3):125-139.

11. Portera CA, Jr., Ho V, Patel SR, Hunt KK, Feig BW, Respondek PM, Yasko AW, et al. Alveolar soft part sarcoma: clinical course and patterns of metastasis in 70 patients treated at a single institution. Cancer. 2001;91(3):585-591.
12. Lieberman PH, Brennan MF, Kimmel M, Erlandson RA, Garin-Chesa P, Flehinger BY. Alveolar soft-part sarcoma. A clinico-pathologic study of half a century. Cancer. 1989;63(1):1-13.

13. Casanova M, Ferrari A, Bisogno G, Cecchetto G, Basso E, De Bernardi B, Indolfi P, et al. Alveolar soft part sarcoma in children and adolescents: A report from the Soft-Tissue Sarcoma Italian Cooperative Group. Ann Oncol. 2000;11(11):1445-1449.

14. BaniHani MN, Al Manasra AR. Spontaneous regression in alveolar soft part sarcoma: case report and literature review. World J Surg Oncol. 2009;7:53. 